\title{
Measurement of the incidence of care-associated adverse events at the Department of Pediatrics of a teaching hospital
}

\author{
Ana Fajreldines, M.D. ${ }^{a}$, Eduardo Schnitzler, M.D. ${ }^{+b}$, Silvio Torres, M.D. ${ }^{c}$, \\ Néstor Panattieri, M.D. ${ }^{d}$ and Marcelo Pellizzari, M.D. ${ }^{a}$
}

\begin{abstract}
Introduction. Adverse events may be detected using different screening tools. In the pediatric population, the Pediatric Trigger Tool looks for triggers in the medical record to detect preventableand non-preventable care-associated harm.

Objective. To measure the incidence of adverse events at the Department of Pediatrics using this tool. To know the types of events and associated outcome measures.

Population and methods. Longitudinal cohort study.

Results. A total of 318 patients were included; mean age: 2.99 years (range: $0-17) ; 164(51.57 \%)$ were girls. There were $11 \%$ of events per 100 admissions, 15.5 events per 1000 patient-days. The most common events were associated with medication use: $48.57 \%(\mathrm{n}=17)$; bacteriology (infections): $42.85 \%(\mathrm{n}=15)$; and care: $8.57 \%$ $(\mathrm{n}=3)$. Nineteen events were mild $(54.28 \%)$, $14(40 \%)$ extended the length of stay (moderate), and $2(5.71 \%)$ required vital support (severe). A total of 168 triggers were detected; 0.53 triggers per patient, 74.4 triggers per 1000 patientdays, and 4.8 triggers per adverse event. In the multivariate logistic regression analysis, the outcome measures associated with the development of adverse events were high-risk medications, female sex, weight, number of transfers within the hospital, and length of stay longer than 5 days.

Conclusions. Using the Pediatric Trigger Tool helped to identify adverse events in hospitalized pediatric patients and guide improvement measures based on associated outcome measures. Key words: Global Trigger Tool, adverse events, hospitalized child.
\end{abstract}

http:/ / dx.doi.org/10.5546/ aap.2019.eng.e106

To cite: Fajreldines A, Schnitzler E, Torres S, Panattieri N, Pellizzari M. Measurement of the incidence of care-associated adverse events at the Department of Pediatrics of a teaching hospital. Arch Argent Pediatr 2019;117(2):e106-e109.
Ana Fajreldines, M.D.:

fajreldinesa@gmail.com

Funding:

None.

Conflict of interest:

None.

Received: 2-2-2018

Accepted: 11-26-2018

\section{INTRODUCTION}

Adverse events occurring in hospital settings are still an unsolved problem that seriously affects patient safety. In pediatrics, treatment options are offered for complex situations that are challenging for health care process reliability. ${ }^{1}$ Baker et al. have reported a $7.5 \%$ incidence of adverse events during the hospitalization of adults. ${ }^{2}$ In children's hospitals, such incidence reaches $9.2 \%$, and adverse events are more common in teaching facilities than in community hospitals. ${ }^{3}$ The most common screening method is the self-report, although it greatly underestimates the actual incidence. ${ }^{4}$

The Global Trigger Tool (GTT) was developed by the Institute for Healthcare Improvement. ${ }^{5}$ It reviews medical records to identify triggers that may be associated with adverse events and has demonstrated to be more sensitive than the self-report technique. ${ }^{6}$

The GTT was adapted to the pediatric population (Pediatric Trigger Tool, PTT) ${ }^{7}$ and has been applied in children's hospitals. Kirkendall et al., ${ }^{8}$ found an incidence of 1.7 triggers per patient, 1 adverse event per 4.6 triggers, and 36.7 events per 100 admissions. In Argentina, Davenport ${ }^{9}$ observed a rate of 26 adverse events per 100 admissions in a sample of 200 patients using the same methodology.

The objectives of this study were to estimate the incidence of adverse events in hospitalized pediatric patients and know the most common types of adverse events and their associated outcome measures using the PTT. 


\section{POPULATION AND METHODS}

This was a longitudinal cohort study that included patients aged 0-17 years who were hospitalized for at least 48 hours. The study was conducted at a tertiary care, teaching hospital that kept electronic medical records. The study period was July 2015-July 2016.

The sample was selected among discharged patients using numbers randomized by Excel (Windows 7). The sample size was estimated according to the rate formula based on a known universe (2000 annual discharges), an alpha error of 0.05 , a $95 \%$ confidence interval (CI), and a $3 \%$ accuracy. The adverse event rate was $40 \%$. Pediatric discharges accounted for $20 \%$ of all discharges. Monthly patient-days were 1100-1300.

All medical record components were reviewed: clinical course, medications, lab tests, diet, specialist consultation, surgical protocols, and discharge summary. Medical record completion was $80-85 \%$ according to the internal monitoring standards of the Joint Commission International (JCI).

In 2014, the PTT was pilot tested in 50 cases; the Kappa coefficient between observers was 0.78 (95\% CI: 0.11-0.97).

Outcome measures were as recommended by the PTT: event rate per 100 admissions, event rate per 1000 patient-days, and rate of admissions with at least 1 adverse event.

The harm scale used in the study was that recommended by the tool. ${ }^{10}$ Harm categories were as follows: $E$, required intervention (mild); F, extended hospitalization (moderate); $G$ permanent harm (severe); $H$ required vital support (severe); I, contributed to or resulted in death (severe).

Patients with 2 or more transfers were those who were taken to or from different hospital wards or where received treatment or underwent procedures in the following units: surgery, cath lab, critical care, etc.

High-risk medications included cancer drugs, electrolyte concentrates, insulin, etc., according to the drug list recommended by the Institute for Safe Medication Practices (ISMP). ${ }^{11}$

The SPSS 19 software, IBM ${ }^{\circledR}$, was used for statistical analysis. The $\chi^{2}$ test was used for dichotomous outcome measures; and the analysis of variance (ANOVA), for continuous outcome measures. A binary logistic regression was done to analyze outcome measure association.

The Institutional Review Board (IRB) approved the study.

\section{RESULTS}

A total of 318 medical records were reviewed. Patients' mean age was 2.99 years (range: 0-17); standard deviation (SD): 6.64; girls: $51.57 \%$ (164); boys: $48.43 \%$ (154). There were 2257 patient-days. Hospitalization was at the general pediatric care unit: 145 (45.6\%); nursery unit: $82(25.8 \%)$; intensive care unit: 65 (20.4\%); neonatal care unit: $26(8.2 \%)$. A total of 35 adverse events were detected; $11 \%$ (10.2-12.6) per 100 admissions, 15.5 per 1000 patientdays, and $8.17 \%$ (95 \% CI: 77.4-82.4) of admissions with at least 1 adverse event.

The most common events were associated with medication use: $48.6 \%(\mathrm{n}=17)$; microbiology (infections): $42.8 \%(n=15)$; and care: $86 \%(n=3)$. No deaths were recorded. Based on the harm classification, 19 events $(54.28 \%)$ were mild, 14 (40\%) extended the length of stay, and $2(5.71 \%)$ were severe and required vital support. A total of 168 triggers were detected; 0.53 triggers per patient, 74.4 triggers per 1000 patient-days, and 4.8 triggers per adverse event. The most common triggers were related to care: 98 (58.3\%); medication use: 45 (26.78 \%); and microbiology: $25(14.88 \%)$; this is consistent with the type of harm observed. The most common adverse events were desaturation and procedure complications, medication use (vomiting), and related to microbiology (infections), with a positive blood culture. The incidence by unit were general pediatric care unit: $145(45.60 \%)$; nursery unit: $82(25.79 \%)$; intensive care unit: $65(20.44 \%)$; neonatal care unit: $26(8.17 \%)$. In relation to the hospitalization area, $0 \%$ corresponded to the nursery unit; $7.69 \%$, to the neonatal care unit; $12.41 \%$, to the general pediatric care unit; and $18.29 \%$, to the intensive care unit.

At the intensive care unit, there were proportionally more events per 100 admissions than at the general hospitalization ward: 18.29 versus 12.41 ( $p=0.05)$.

The extended length of stay in days was counted as of the day of the event onset. The mean extended length of stay was 3.14 days.

The outcome measures associated with the development of adverse events were high-risk medications, weight, female sex, number of transfers within the hospital, and a length of stay longer than 5 days (Table 1).

\section{DISCUSSION}

The studies that focused on this topic, like the ones by Stockwell et al. ${ }^{12}$ and Kirkendall et al., ${ }^{8}$ found a rate of 40 and 36.7 adverse events per 
100 admissions, respectively. Kirkerdall et al. ${ }^{8}$ reported a higher frequency of surgery-related events (39\%), whereas in our study, medication use (54\%) was the main cause. At our hospital, the medication process did not have an advanced support system but an electronic system with manual security barriers.

The study by Takata et al., ${ }^{13}$ showed more medication-related adverse events than other publications. Their study analyzed more than 900 patients and found 11.11 adverse events related to medications per 100 patients, which is consistent with our data in terms of relevance. Stroupe et al., ${ }^{14}$ in a Canadian study that used the Global Assessment of Pediatric Patient Safety (GAPPS) tool, ${ }^{15}$ found lower rates per 100 cases than in our study and, than in the other studies mentioned here: $4.87 \%$ of adverse events.

Davenport et al. ${ }^{9}$ detected 26 adverse events per 100 admissions and 1.4 triggers per patient, with harm and trigger rates higher than in our study (0.53 triggers per patient and 11 adverse events per 100 admissions in our study). Such difference may be explained by the inclusion of patients who required primary care and were hospitalized at the general pediatric care and nursery units. However, according to the methodology, every hospitalized patient had to be included in the sample so that it would be representative of the total discharges.

The extended length of stay due to careassociated harm was also observed by other authors, like Palacios Barahona et al., ${ }^{16}$ Kaushal et al., ${ }^{17}$ and Hug et al. ${ }^{18}$

\section{CONCLUSIONS}

Although this is one of the first experiences using the PTT in Argentina, and considering the varied bibliography on this topic and the widespread results in terms of harm in pediatric patients, it is believed that this tool will be an opportunity to redesign processes and improve patient safety.
High-risk medication use, weight, female sex, number of transfers within the hospital, and a length of stay longer than 5 days were the outcome measures associated with adverse events.

\section{REFERENCES}

1. Porter ME. What is Value in Health Care? N Engl J Med. 2010; 363(26):2477-81.

2. Baker GR, Norton PG, Flintoft V, Blais R, et al. The Canadian Adverse Events Study: the incidence of adverse events among hospital patients in Canada. CMAJ. 2004; 170(11):1678-86.

3. Matlow AG, Baker GR, Flintoft V, CochraneD, et al. Adverse events among children in Canadian hospitals: the Canadian Pediatric Adverse Events Study.CMAJ. 2012;184(13):E70918.

4. AHRQ QI Research Version 5.0, Pediatric Quality Indicators \#19, Technical Specifications. Pediatric Patient Safety for Selected Indicators. [Accessed on: December $3^{\text {rd }}$, 2017]. Available at: www.qualityindicators.ahrq. gov / Downloads / Modules / PDI / V50 / TechSpecs / PDI_19_Pediatric_Safety_for_Selected_Indicators.pdf.

5. Griffin FA, ResarRK. IHIGlobal TriggerTool for Measuring Adverse Events. $2^{\text {nd }}$ ed. IHI Innovation Series white paper. Cambridge, MA: Institute for Healthcare Improvement; 2009.

6. Classen DC, Resar R, Griffin F, Federico F, et al. Global Trigger Tool shows that adverse events in hospitals may be ten times greater than previously measured. Health Aff (Millwood). 2011; 30(4):581-9.

7. NHS Institute for Innovation and Improvement. Paediatric Trigger. User guide. [Accessed on: December $\left.3^{\text {rd }}, 2017\right]$. Available at: https://bmjopen.bmj.com/content/ suppl/2014/07/03/bmjopen-2014-005066.DC1/bmjopen2014-005066supp2.pdf

8. Kirkendall ES, Kloppenborg E, Papp J, White D, et al. Measuring adverse events and levels of harm in pediatric inpatients with the Global Trigger Tool. Pediatrics. 2012; 130(5):e1206-14.

9. Davenport MC, Domínguez PA, Ferreira JP, Kannemann $\mathrm{AL}$, et al. Detección de eventos adversos en pacientes pediátricos hospitalizados mediante la herramienta de disparadores globales Global Trigger Tool. Arch Arg Pediatr. 2017; 115(4):357-63.

10. Nacional Coordinating Council for Medication Error Prevention. Taxonomy of Medication Error Now Available. [Accessed on: August 2016]. Available at: http://www. nccmerp.org/taxonomy-medication-errors-now-available.

11. InstituteforSafe Medication Practices. High alert medication lists. [Accessed on: August2016]. Availableat:https:/ / www. ismp.org/tools/highalertmedicationlists.asp.

TABLE 1. Outcome measures associated with adverse events

\begin{tabular}{lccc}
\hline Outcome measure & OR $_{\mathrm{AJ}}$ & $\mathbf{9 5} \% \mathbf{C I}$ & $p$ value \\
\hline Patients on high-risk medications & 2.13 & $1.24-5.12$ & 0.004 \\
Length of stay longer than 5 days & 1.84 & $0.11-2.24$ & 0.05 \\
More than one transfer & 2.06 & $1.54-6.76$ & 0.04 \\
Weight & 2.89 & $1.61-4.26$ & 0.01 \\
Assisted mechanical ventilation & 0.55 & $0.41-1.26$ & $0.4(\mathrm{NS})$ \\
Female sex & 1.78 & $1.24-2.49$ & 0.01 \\
\hline
\end{tabular}

$\mathrm{OR}_{\text {adj }}$ : adjusted odds ratio; CI: confidence interval; NS: not significant. 
12. Stockwell DC, Bisarya H, Classen D, Kirkendall ES, et al. A trigger tool to detect harm in pediatric inpatient settings. Pediatrics. 2015; 135(6):1036-42.

13. Takata GS, Mason W, Taketomo C, Logsdon T, et al. Development, testing, and findings of a pediatric-focused trigger tool to identify medication-related harm in US children's hospitals. Pediatrics. 2008; 121(4):e927-35.

14. Stroupe LM, Patra KP, Dai Z, Lancaster J, et al. Measuring Harm in Hospitalized Children via a Trigger Tool. J Pediatr Nurs. 2017; S0882-5963(16)30295-0.

15. Landrigan CP, Stockwell D, Toomey SL, Loren S, et al. Performance of the Global Assessment of Pediatric Patient
Safety (GAPPS) Tool. Pediatrics. 2016; 137(6):e20154076.

16. Palacios-Barahona AU, Bareño-Silva J. Factores asociados a eventos adversos en pacientes hospitalizados en una entidad de salud en Colombia. Rev CES Med. 2012;26(1):1928.

17. Kaushal R, Bates DW, Franz C, Soukup JR, et al. Costs of adverse events in intensive care units. Crit Care Med. 2007; 35(11):2479-83.

18. Hug BL, Keohane C, Seger DL, Yoon C, et al. The costs of adverse drug events in community hospitals. Jt Comm J Qual Patient Saf. 2012; 38(3):120-6. 